\title{
Neuropathic Pain after Adolescent Idiopathic Scoliosis Correction Surgery
}

\author{
Mohd Shahnaz Hasan ${ }^{1}$, Khean Jin Goh ${ }^{2}$, Hing Wa Yip ${ }^{1}$, Siti Mariam Mohamad ${ }^{3}$, Teik Seng Chan ${ }^{3}$, \\ Kok Ian Chong ${ }^{3}$, Amber Haseeb ${ }^{3}$, Chee Kidd Chiu ${ }^{3}$, Chris Chan Yin Wei ${ }^{3}$, Mun Keong Kwan ${ }^{3}$ \\ ${ }^{I}$ Department of Anesthesiology, Faculty of Medicine, University of Malaya, Kuala Lumpur, Malaysia \\ ${ }^{2}$ Department of Medicine, Faculty of Medicine, University of Malaya, Kuala Lumpur, Malaysia \\ ${ }^{3}$ Department of Orthopaedic Surgery, National Orthopaedic Centre of Excellence for Research and Learning, Faculty of Medicine, \\ University of Malaya, Kuala Lumpur, Malaysia
}

\begin{abstract}
Study Design: Prospective study.
Purpose: To investigate the prevalence and the associated risk factors of chronic neuropathic pain symptoms using painDETECT questionnaire in adolescent idiopathic scoliosis (AIS) patients who underwent posterior spinal fusion (PSF) surgery.

Overview of Literature: Post-lumbar surgery syndrome is a disease entity that describes neuropathic pain following spinal surgery. However, few studies have investigated the prevalence and risk factors for neuropathic pain in pediatric population undergoing corrective spinal surgery.

Methods: Forty AIS patients were recruited. Demographic, preoperative, and postoperative data were recorded. The magnitude and characteristics of postoperative pain were assessed using the painDETECT questionnaire through telephone enquiries at intervals of $2,6,12$, and 24 weeks. Statistical analyses were followed by Pearson correlation test to determine the relationship between pain scores at 6,12 , and 24 weeks with the risk factors.

Results: Based on the painDETECT questionnaire, $90 \%$ of the patients had nociceptive pain, and $10 \%$ had a possible neuropathic pain component at 2 weeks postoperatively as per a mean painDETECT score of $7.1 \pm 4.5$. Assessments at 6,12 , and 24 weeks showed that no patients had neuropathic pain with painDETECT scores of $4.4 \pm 3.2,2.9 \pm 2.9$, and $1.5 \pm 2.0$, respectively. There was a significant correlation between total postoperative morphine use during 48 hours after the surgery and a tendency to develop neuropathic pain $(p=0.022)$.

Conclusions: Chronic neuropathic pain was uncommon in AIS patients who had undergone PSF surgery. Higher opioid consumption will increase the possibility of developing chronic neuropathic pain.
\end{abstract}

Keywords: Neuropathic; Scoliosis; Surgery; PainDETECT

\section{Introduction}

Persistent or chronic pain is being increasingly reported in different surgical procedures, such as spinal surgery, amputation, mastectomy, thoracotomy, and craniotomy [1]. Based on the diagnostic criteria of the International Asso-

Received Jan 9, 2020; Revised Jun 25, 2020; Accepted Jun 25, 2020

Corresponding author: Chris Chan Yin Wei

Department of Orthopaedic Surgery, National Orthopaedic Centre of Excellence for Research and Learning (NOCERAL), Faculty of Medicine, University of Malaya, 50603 Kuala Lumpur, Malaysia

Tel: +60-128830301, Fax: +60-379494642, E-mail: chrnat01@um.edu.my 
ciation for the Study of Pain (IASP), chronic postsurgical pain is defined as "pain persisting for at least 3 months after surgery, pain that is different from preoperative pain, and pain caused by other conditions such as continuing malignancy or chronic infection should be excluded." Such persistent pain can be neuropathic in nature [2].

The definition of neuropathic pain by the IASP is pain caused by a lesion or disease of the somatosensory system. As per research, $10 \%$ of those aged $\geq 30$ years had neuropathic pain $[3,4]$. Patients who experience neuropathic pain usually develop numbness, tingling, electric-shocklike sensations, burning pain, allodynia, hypersensitivity to heat and cold, cutaneous hyperalgesia, and pain attack [5].

Corrective spinal surgery for scoliosis is a major operative procedure and is often linked with severe perioperative pain. Failed back surgery syndrome or post-lumbar surgery syndrome is a disease entity that describes neuropathic pain after spinal surgery and is frequently observed in adults. However, few studies have investigated the prevalence and risk factors for neuropathic pain in a pediatric population undergoing corrective spinal surgery.

This study aimed to determine the prevalence of chronic neuropathic pain symptoms in adolescent idiopathic scoliosis (AIS). Moreover, the risk factors that contribute to the development of chronic neuropathic pain were also evaluated.

\section{Materials and Methods}

This observational study was approved by the Medical Research Ethics Committee of University of Malaya Medical Centre (ref no., MECID-2015-10-1703). Written informed consent was obtained from either patients or their parents. Patients who had undergone elective posterior spinal fusion (PSF) surgery from September 2015 to December 2015 were recruited. The inclusion criteria were as follows: age 10-21 years and American Society of Anesthesiologists physical status I to II. As per the exclusion criteria, patients with non-idiopathic scoliosis, known psychological disorder, diabetes mellitus, revision surgery, and those with a communication barrier were excluded. Patients on preoperative long-term opioids, sedatives, or anticonvulsants treatment were also excluded. The anesthesia protocol, surgery protocol, and pain management regime were standardized for all the patients. Total 40 patients were identified and enrolled in the study.

\section{Anesthesia protocol}

In the operating room, standard monitoring with pulse oximetry, electrocardiography, and automated noninvasive blood pressure were applied before anesthesia administration. Bispectral index (BIS) device was used for monitoring the depth of anesthesia. The patient was given intravenous (IV) propofol $2.5 \mathrm{mg} / \mathrm{kg}$, IV target controlled infusion (TCI) of remifentanil $5 \mathrm{ng} / \mathrm{mL}$, and IV rocuronium $1 \mathrm{mg} / \mathrm{kg}$ to facilitate endotracheal intubation. Anesthesia was maintained with desflurane and TCI remifentanil $2-8 \mathrm{ng} / \mathrm{mL}$ for the maintenance of BIS value of 40-60 during surgery. All patients received $0.2 \mathrm{mg} / \mathrm{kg}$ of IV morphine 45 minutes and $1 \mathrm{mcg} / \mathrm{kg}$ of IV fentanyl 10 minutes before the end of surgery. IV dexamethasone $4 \mathrm{mg}$ and IV ondansetron $4 \mathrm{mg}$ were administered as prophylactics for postoperative nausea and vomiting.

\section{Surgical protocol}

All the patients underwent PSF using alternate level pedicle screw configuration with three to four consecutive pedicle screws at lower end and upper end as base anchor and as proximal anchor. However, in stiff curves and curves $>90^{\circ}$, more screws were placed to assist correction and enhance fusion. Facetectomies were performed to increase the spinal flexibility and to facilitate spinal fusion. No Ponte osteotomies were performed. Reduction was performed using translation method and direct vertebral rotation. Fusion was augmented using autogenous local bone graft obtained from spinous processes, transverse processes, and decorticated lamina of each instrumented vertebrae. Cell salvage autologous blood recovery system was used in all cases (Haemonetics Cell Saver 5+). Topical hemostatic agents were not used in any cases. A deep subfascial drain was inserted for all patients.

\section{Postoperative pain management regime}

Prior to skin closure, $2 \mathrm{mg} / \mathrm{kg}$ bupivacaine diluted to a volume of $25 \mathrm{~mL}$ was infiltrated subcutaneously. In the recovery room, all the patients received patient-controlled analgesia (PCA) IV morphine with the following preparation: PCA bolus of $1 \mathrm{mg}$ morphine with a 5-minute lockout interval and 4-hour dose limit of $20 \mathrm{mg}$ morphine. PCA morphine was provided for at least 48 hours postoperatively and will be discontinued once consumption was 
$<5 \mathrm{mg}$ within 24 hours. Oral analgesia in the form of celecoxib (Celebrex) capsule $200 \mathrm{mg}$ once/twice daily and oral paracetamol 500-1,000 mg 6 hourly were commenced as soon as the patient was able to tolerate oral intake. After the discontinuation of PCA morphine, breakthrough pain was managed with immediate-release oxycodone hydrochloride (OxyNorm) capsule $5 \mathrm{mg}$.

\section{Postoperative rehabilitation protocol}

Drain was removed between 12 and 24 hours after the surgery with drainage of a maximum of $200 \mathrm{~mL}$ at the time of removal. Dressing was changed, and the urinary catheter was removed at the same time. The patient was then allowed to sit at the edge of the bed and encouraged to sit up and ambulate as tolerated (except in patients with severe postural dizziness/nausea).

\section{Data collection}

Demographic, preoperative, and postoperative data were recorded. The total PCA morphine usage and the magnitude of postoperative pain was charted using the Visual Analog Scale (VAS) pain score 12 hourly for up to 48 hours. Assessment of the pain score using the painDETECT questionnaire after discharge was performed via telephone enquiry at 2 weeks, 6 weeks, 3 months, and 6 months after the surgery. The painDETECT questionnaire is a validated screening tool for back pain that includes seven questions that address the characteristics and quality of neuropathic pain symptoms. If the painDETECT score is $\leq 12$, the pain is likely to be nociceptive in origin ( $<15 \%$ to be neuropathic), a score of $12-18$ indicates ambiguity (can be nociceptive or neuropathic or mixture of both), and a score $>18$ indicates that the pain is likely to be neuropathic in nature.

\section{Power analysis, sample size, and statistical analyses}

The present study is the continuous observation from a previous study that examined acute postoperative pain [6]. A pilot study that involved 10 subjects was performed to calculate the sample size for this study. Considering $5 \%$ marginal error and $80 \%$ study power, a statistical power analysis indicated that a minimum sample size of 36 subjects was needed to detect the mean of the outcome variables.
The data from this investigation were entered and analyzed using the IBM SPSS ver. 23.0 (IBM Corp., Armonk, NY, USA) to make inferences and draw robust conclusions. In brief, descriptive statistical analysis of the sociodemographic characteristics was initially performed to evaluate data distribution, normality, and homogeneity. Descriptive analysis included frequency and percentage distribution for the categorical variables as well as the mean and standard deviation for numerical variables. Statistical analyses were performed using the Pearson correlation test to determine the relationship between the pain score at 6 months and the risk factors. Repeated measure analysis of variance was used to identify any differences in the mean pain score based on time ( 2 weeks, 6 weeks, 3 months, and 6 months). A $p$-value $<0.05$ was considered to indicate statistical significance.

\section{Results}

Forty patients with AIS who underwent corrective surgery were enrolled. The demographic data shown in Table 1 depicts that the average patient age was $15.7 \pm 3.6$ years; $10 \%$ of the population comprised male subjects. Most patients were Chinese (72.5\%) and had Lenke type 1 (40\%). The average Cobb's angle of the patients was $66.5^{\circ} \pm 16.0^{\circ}$. The average wound size, operation time, total blood loss, total fusion level, and number of days in the hospital were $28.9 \pm 5.7 \mathrm{~cm}, 162.4 \pm 59.4$ minutes, $992.3 \pm 559.1 \mathrm{~mL}$, $9.8 \pm 2.2$, and $3.8 \pm 0.7$ days, respectively.

The painDETECT questionnaire was used for screening for the presence of neuropathic pain component. Based on the final score from the painDETECT questionnaire, $90 \%$ of the patients were in the nociceptive pain category (unlikely to be neuropathic in nature), and the remaining $10 \%$ were in the unclear category (a neuropathic pain component can be present) after 2 weeks post-surgery. Further assessments performed at 6 weeks, 3 months, and 6 months revealed that none of the patients were likely to have neuropathic pain (Table 2).

Repeated measures analysis performed to determine the mean difference of the final painDETECT score based on the different time intervals found an overall $p$-value of $<0.001$. The analysis also showed a decreasing mean score over the measured time points (Table 2). Pairwise comparison method was further performed and showed significant difference between week 2-week $6(p<0.001)$, week 2 -month $3(p<0.001)$, week 2 -month $6(p<0.001)$, 
Table 1. Demographic data of the patients

\begin{tabular}{|c|c|}
\hline Characteristic & Value \\
\hline Age (yr) & $15.7 \pm 3.6$ \\
\hline \multicolumn{2}{|l|}{ Race } \\
\hline Chinese & $29(72.5)$ \\
\hline Malay & $4(10.0)$ \\
\hline Indian & $3(7.5)$ \\
\hline Others & $4(10.0)$ \\
\hline \multicolumn{2}{|l|}{ Gender } \\
\hline Male & $4(10.0)$ \\
\hline Female & $36(90.0)$ \\
\hline Weight (kg) & $46.9 \pm 10.3$ \\
\hline Height (cm) & $156.3 \pm 8.2$ \\
\hline Body mass index $\left(\mathrm{kg} / \mathrm{m}^{2}\right)$ & $19.2 \pm 3.8$ \\
\hline \multicolumn{2}{|l|}{ Lenke types } \\
\hline 1 & $16(40.0)$ \\
\hline 2 & $7(17.5)$ \\
\hline 3 & $3(7.5)$ \\
\hline 4 & $2(5.0)$ \\
\hline 5 & $8(20.0)$ \\
\hline 6 & $4(10.0)$ \\
\hline Cobb angle $\left({ }^{\circ}\right)$ & $66.5 \pm 16.0$ \\
\hline \multicolumn{2}{|l|}{ Operative data } \\
\hline Wound size $(\mathrm{cm})$ & $28.9 \pm 5.7$ \\
\hline Operation time (min) & $162.4 \pm 59.4$ \\
\hline Blood loss (mL) & $992.3 \pm 559.1$ \\
\hline Fusion level & $9.8 \pm 2.2$ \\
\hline Hospital stay (day) & $3.8 \pm 0.7$ \\
\hline
\end{tabular}

Values are presented as mean \pm standard deviation or number (\%).

Table 2. Distribution of final painDETECT score of each time points

\begin{tabular}{|c|c|c|c|c|}
\hline \multirow{2}{*}{ Time } & \multirow{2}{*}{$\begin{array}{c}\text { Mean } \pm \text { standard } \\
\text { deviation }\end{array}$} & \multicolumn{3}{|c|}{ No. of neuropathic pain component (\%) } \\
\hline & & Nociceptive & Unclear & Neuropathic \\
\hline 2 wk & $7.1 \pm 4.5$ & $36(90.0)$ & $4(10.0)$ & 0 \\
\hline 6 wk & $4.4 \pm 3.2$ & $40(100.0)$ & 0 & 0 \\
\hline $3 \mathrm{mo}$ & $2.9 \pm 2.9$ & $40(100.0)$ & 0 & 0 \\
\hline $6 \mathrm{mo}$ & $1.5 \pm 2.0$ & $40(100.0)$ & 0 & 0 \\
\hline
\end{tabular}

week 6-month 3 ( $p=0.002)$, week 6-month $6(p<0.001)$, and month 3 -month $6(p<0.001)$ (Table 3$)$.

Correlation between different risk factors and painDETECT score obtained at 6,12 , and 24 weeks post-surgery
Table 3. Comparison of final score between different time points using painDETECT questionnaire

\begin{tabular}{lcc} 
Comparison & Mean difference (95\% CI) & $p$-value \\
\hline Week 2-week 6 & $2.6(0.976-4.724)$ & $<0.001$ \\
Week 2-month 3 & $4.2(2.354-6.046)$ & $<0.001$ \\
Week 2-month 6 & $5.6(3.678-7.422)$ & $<0.001$ \\
\hline Week 6-month 3 & $1.6(0.445-2.705)$ & 0.002 \\
\hline Week 6-month 6 & $2.9(1.634-4.216)$ & $<0.001$ \\
\hline Month 3-month 6 & $1.4(0.595-2.105)$ & $<0.001$ \\
\hline
\end{tabular}

Pairwise comparison was applied with $95 \% \mathrm{Cl}$ adjustment by Bonferroni correction.

$\mathrm{Cl}$, confidence interval.

Table 4. Correlation between painDETECT score at 6, 12, 24 weeks and the risk factors

\begin{tabular}{|c|c|c|c|}
\hline Risk factor & Mean $\pm S D$ & $\begin{array}{c}r \text { (correlation } \\
\text { coefficient) }\end{array}$ & $p$-value \\
\hline \multicolumn{4}{|l|}{ PainDETECT score at 6 wk } \\
\hline Total morphine used at $48 \mathrm{hr}$ (mg) & $27.2 \pm 21.6$ & 0.295 & 0.065 \\
\hline \multicolumn{4}{|l|}{ PainDETECT score at 12 wk } \\
\hline Postoperative pain score & $6.5 \pm 1.9$ & -0.342 & 0.031 \\
\hline Total morphine used at $48 \mathrm{hr}$ (mg) & $27.2 \pm 21.6$ & 0.505 & 0.001 \\
\hline \multicolumn{4}{|l|}{ PainDETECT score at 24 wk } \\
\hline Total morphine used at $48 \mathrm{hr}(\mathrm{mg})$ & $27.2 \pm 21.6$ & 0.36 & 0.022 \\
\hline \multicolumn{4}{|c|}{$\begin{array}{l}\text { PainDETECT score at } 6 \text { weeks: mean } \pm S D, 4.4 \pm 3.2 ; 12 \text { weeks: mean } \pm S D, 2.9 \pm 2.9 \text {; } \\
\text { and } 24 \text { weeks: mean } \pm S D, 1.5 \pm 2.0 \text {. There were no significant correlations be- } \\
\text { tween other risk factors such as wound size, preoperative Cobb's angle, correc- } \\
\text { tion rate, number of fusion levels, surgery duration, and total blood loss. } \\
S D \text {, standard deviation. }\end{array}$} \\
\hline
\end{tabular}

was done (Table 4). Total morphine usage at 48 hours post-surgery showed significant positive correlation with the painDETECT scoring at all time points. Postoperative pain score showed significant negative correlation with painDETECT score at 12 weeks but not at 6 and 24 weeks. Other risk factors such as wound size, preoperative Cobb's angle, correction rate, duration of surgery, fusion level, and total blood loss intraoperatively did not show any significant correlations with the painDETECT score at all time points (Table 4 ).

The mean plot versus time intervals was performed to determine the trend of pain score over time. The painDETECT score consists of seven different types of pain, including burning, tingling or prickling, light touch (allodynia), electric shock, temperature, numbness, and pressure. From the mean plot, all types of sensation showed reduction in trend during the 6-month period (Fig. 1). 


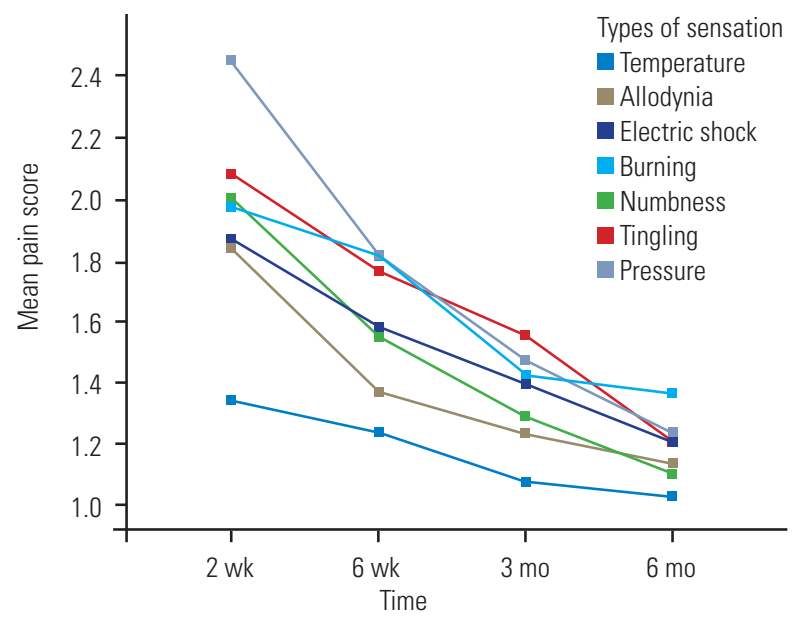

Fig. 1. The mean score over time of the different types of pain in painDETECT questionnaire.

\section{Discussion}

It is known that surgical trauma can induce hyperalgesia, a cause of post-surgery neuropathic pain. During the surgery, peripheral pain signals are transmitted through peripheral sensory fibers, mainly $A \beta$ type fibers and $C$ fibers, to reach the central nervous system for integration. A complex system of adaptive processes is activated when the surgical stimuli is maintained. The neurotransmitters involved in these signals modulation are mainly glutamate and substance P. For achieving an effective analgesic effect and prevent neuropathic pain transformation, the production and generation of action potentials in $\mathrm{A}$ and $\mathrm{C}$ fibers need to be diminished. Analgesia can be achieved by inhibiting the transmission of pain information along the spine [7].

The diagnosis of neuropathic pain is graded into possible, probable, and definite, based on history, physical examination, and diagnostic investigation [8]. However, in the epidemiological setting, screening tools, such as the painDETECT questionnaire and Neuropathic Pain Screening Questionnaire (NePSQ), were developed and validated for screening neuropathic pain symptoms [9]. The painDETECT questionnaire was developed initially to screen for neuropathic pain in back pain patients; however, it was useful for other causes of neuropathic pain with a sensitivity and specificity of $85 \%$ and $80 \%$, respectively [10]. In a study on elderly chronic low back pain (LBP) patients that utilized two different questionnaires, the NePSQ and the painDETECT questionnaire, it was concluded that the painDETECT questionnaire was more suitable than NePSQ for screening neuropathic pain in chronic LBP [11]. As such, the painDETECT questionnaire was used in this study to identify subjects with chronic neuropathic pain symptoms.

These neuropathic pain screening tools have been validated in adults [12]; however, their evaluation and use in children are limited. Numerous studies have used the painDETECT questionnaire to assess neuropathic pain in adolescent and pediatric patients with oncological disease [13]. It has been validated by the developer down to 14 years that supports the capability of adolescents to understand the pain descriptors used [14]. It has $83 \%$ positive predictive value to differentiate neuropathic pain from non-neuropathic pain [15]. The mean age of our study population was $15.7 \pm 3.6$ years, and all of them were capable of understanding the pain descriptors. This makes the painDETECT questionnaire a suitable self-reporting tool for data collection via telephone interview.

Few studies have examined chronic neuropathic pain in pediatric subjects who have undergone scoliosis surgery. From our data, we observed that the prevalence of chronic neuropathic pain is very low in scoliosis surgery. All patients experienced minimal pain that not significant enough to be defined as neuropathic in nature as early as 6 weeks after the surgery. Thus, the pain that the patients experienced may be described as "persistent postsurgical pain [1]."

There was a significant correlation between total postoperative morphine use during 48 hours after the surgery and the tendency to develop neuropathic pain. More severe the pain during the early perioperative period, greater the opioid consumption through the PCA route. In other words, the severity of perioperative pain will determine the likelihood of developing chronic neuropathic pain. This is in concordance with several studies in adults and children, although the data in children are less comprehensive [16]. Following scoliosis and pectus surgery, the higher pain score recorded during the first 72 hours predicts slower recovery and higher pain score at 4 months and 12 months [17]. In the study by Julien-Marsollier et al. [18], persistent preoperative pain and total morphine consumption on day 1 were associated with the occurrence of chronic neuropathic pain. Similarly, Chidambaran et al. [19] also reported that acute postoperative pain on day 1 and 2 after spinal surgery is a significant predictor of persistent and chronic pain.

Multiple case reports have shown evidence of the development of opioid induced hyperalgesia in patients 
receiving higher doses of morphine. Most of these studies involve systemic or intrathecal morphine administration. It is postulated that hyperalgesia is caused by the metabolite morphine-3-glucoronide causing neuroexcitation [15]. Therefore, the concept of multimodal analgesia should be emphasized to reduce the amount of morphine usage while producing similar analgesic efficacy during the perioperative period, thus reducing the development of neuropathic pain in the future.

From our study protocol, we designed a multimodal analgesia approach during the perioperative period. The analgesic regimen consists of the combination of TCI remifentanil, IV fentanyl, IV morphine, and local anesthetic infiltration at the incision site intraoperatively with PCA morphine and oral paracetamol, celecoxib, and oxycodone during the postoperative period. Nonetheless, four patients still experienced some form of pain during the first 2 postoperative weeks that was categorized as ambiguous (mixture of nociceptive and neuropathic nature).

Some studies have used anti-neuropathics, such as pregabalin, perioperatively in adult male lumbar spinal fusion surgery that effectively reduced the opioid usage with similar efficacy in pain control [20]. Pregabalin has demonstrated an analgesic and anti-hyperalgesic effect on postoperative lumbar disc hernia surgery [21]. Therefore, the perioperative use of pregabalin should be considered for neuropathic treatment and as an adjunct for reducing opioid consumption [22]. Again, the data in pediatric population is sparse and the anti-neuropathic use is mainly confined to chronic neuropathic pain, epilepsy, or dysautonomic crisis [23].

Surgical trauma can induce hyperalgesia leading to acute neuropathic pain. Hence, we evaluated different surgery-related risk factors, such as surgical wound size, preoperative Cobb's angle, number of fusion levels, postoperative Cobb's angle correction rate, duration of surgery, and total blood loss. These factors were used as surrogates of the degree of surgery-induced tissue trauma and showed no association with neuropathic pain.

Our study also examined different time intervals after the surgery. From 6 weeks onward, all the patients continued to experience some pain that was not significant enough to be categorized under the neuropathic category. Further analysis based on other subtypes of pain experienced by patients also demonstrated a lowering trend with time (Fig. 1). To explain this phenomenon, we analyzed the adult population data and compared total morphine use after 48 hours of surgery between the adult and pediatric groups. For adult populations, the PCA morphine usage was more, around $100 \mathrm{mg}$ within 48 hours of the surgery [20], than that in our study (mean, $27.2 \mathrm{mg}$ ); the difference was approximately 4 fold. The relatively low opioid consumption was a result of the multimodal analgesia design that was adopted from the beginning of the study. Therefore, the prevalence of neuropathic pain in our study was low. A recent study by Julien-Marsollier et al. [18] also demonstrated that the total morphine consumption on postoperative day 1 was associated with the occurrence of chronic neuropathic pain.

Our study has certain limitations. First, this observational study was the continuation of a previous study. The sample size might not adequately represent the population. A larger sample size may be required. Owing to the relatively small sample size, some risk factors are yet to be determined.

The painDETECT questionnaire used in our study could only enable a diagnosis of "probable" neuropathic pain. The questionnaire was completed via phone call interview that did not involve a clinical examination. Diagnostic tests are required to confirm the diagnosis of "definite" neuropathic pain. Diagnostic tests can help in locating the lesion or diseases of the somatosensory nervous system; however, these tests do not establish the causality.

Neuropathic pain can be acute in nature and may develop in the early postoperative period. The severity of preoperative pain has been identified as a risk factor for the development of chronic pain in pediatric patients, particularly in scoliosis surgery $[24,25]$. The painDETECT screening questionnaire data should be collected preoperatively and every day after the surgery.

Neuropathic pain is very debilitating, especially if it develops at a younger age. It has a considerable impact not only on patients, but also on the health care systems and society. Hence, it should be aggressively treated. More studies on neuropathic pain have been conducted in the previous decades that have improved our knowledge and treatment modalities; we believe that the best strategy would be to focus on prevention. We found that increased perioperative pain and opioid consumption raise the possibility of developing chronic neuropathic pain; therefore, a multimodal approach should be considered to reduce the perioperative opioid usage. 


\section{Conclusions}

Chronic neuropathic pain was uncommon in AIS patients who had undergone PSF surgery. Higher opioid consumption will increase the possibility of developing chronic neuropathic pain.

\section{Conflict of Interest}

No potential conflict of interest relevant to this article was reported.

\section{References}

1. Lavand'homme P. The progression from acute to chronic pain. Curr Opin Anaesthesiol 2011;24:54550.

2. Steegers MA, Snik DM, Verhagen AF, van der Drift MA, Wilder-Smith OH. Only half of the chronic pain after thoracic surgery shows a neuropathic component. J Pain 2008;9:955-61.

3. Yawn BP, Wollan PC, Weingarten TN, Watson JC, Hooten WM, Melton LJ 3rd. The prevalence of neuropathic pain: clinical evaluation compared with screening tools in a community population. Pain Med 2009;10:586-93.

4. Bouhassira D, Lanteri-Minet M, Attal N, Laurent B, Touboul C. Prevalence of chronic pain with neuropathic characteristics in the general population. Pain 2008;136:380-7.

5. Rehm SE, Koroschetz J, Gockel U, et al. A crosssectional survey of 3035 patients with fibromyalgia: subgroups of patients with typical comorbidities and sensory symptom profiles. Rheumatology (Oxford) 2010;49:1146-52.

6. Kwan MK, Chiu CK, Chan TS, et al. Trajectory of postoperative wound pain within the first 2 weeks following posterior spinal fusion surgery in adolescent idiopathic scoliosis patients. Spine (Phila Pa 1976) 2017;42:838-43.

7. Finnerup NB, Otto M, McQuay HJ, Jensen TS, Sindrup SH. Algorithm for neuropathic pain treatment: an evidence based proposal. Pain 2005;118:289-305.

8. Finnerup NB, Haroutounian S, Kamerman P, et al. Neuropathic pain: an updated grading system for research and clinical practice. Pain 2016;157:1599-606.

9. Ogawa S. Development of new screening question- naire to identify neuropathic components in Japanese patients with chronic pain. Pain Clin 2010;31:118794.

10. Bennett MI, Attal N, Backonja MM, et al. Using screening tools to identify neuropathic pain. Pain 2007;127:199203.

11. Sakai Y, Ito K, Hida T, Ito S, Harada A. Neuropathic pain in elderly patients with chronic low back painand effects of pregabalin: a preliminary study. Asian Spine J 2015;9:254-62.

12. Bendinger T, Plunkett N. Measurement in pain medicine. BJA Educ 2016;16:310-5.

13. Acquazzino M, Dasgupta M, Hoffmann R, Browning M, Brandow A. Patient-reported prevalence of neuropathic pain in adolescent and young adult oncology patients. J Clin Oncol 2016;34(15 Suppl):10577.

14. Freynhagen R, Baron R, Gockel U, Tolle TR. PainDETECT: a new screening questionnaire to identify neuropathic components in patients with back pain. Curr Med Res Opin 2006;22:1911-20.

15. Lee M, Silverman SM, Hansen H, Patel VB, Manchikanti L. A comprehensive review of opioid-induced hyperalgesia. Pain Physician 2011;14:145-61.

16. Williams G, Howard RF, Liossi C. Persistent postsurgical pain in children and young people: prediction, prevention, and management. Pain Rep 2017;2:e616.

17. Rabbitts JA, Zhou C, Groenewald CB, Durkin L, Palermo TM. Trajectories of postsurgical pain in children: risk factors and impact of late pain recovery on long-term health outcomes after major surgery. Pain 2015;156:2383-9.

18. Julien-Marsollier F, David R, Hilly J, Brasher C, Michelet D, Dahmani S. Predictors of chronic neuropathic pain after scoliosis surgery in children. Scand J Pain 2017;17:339-44.

19. Chidambaran V, Ding L, Moore DL, et al. Predicting the pain continuum after adolescent idiopathic scoliosis surgery: a prospective cohort study. Eur J Pain 2017;21:1252-65.

20. Kim JC, Choi YS, Kim KN, Shim JK, Lee JY, Kwak YL. Effective dose of peri-operative oral pregabalin as an adjunct to multimodal analgesic regimen in lumbar spinal fusion surgery. Spine (Phila Pa 1976) 2011;36:428-33.

21. Canos A, Cort L, Fernandez Y, et al. Preventive analgesia with pregabalin in neuropathic pain from "failed back surgery syndrome": assessment of sleep quality 
and disability. Pain Med 2016;17:344-52.

22. Taylor CP. The biology and pharmacology of calcium channel alpha2-delta proteins Pfizer Satellite Symposium to the 2003 Society for Neuroscience Meeting. Sheraton New Orleans Hotel, New Orleans, LA November 10, 2003. CNS Drug Rev 2004;10:183-8.

23. Portniagin IV, Dunayevskiy IV, Strelnikov AA. 672 Pregabalin: an efficacious drug for neuropathic pain management in children. Eur J Pain 2009;13(Suppl 1):S194.
24. Landman Z, Oswald T, Sanders J, Diab M; Spinal Deformity Study Group. Prevalence and predictors of pain in surgical treatment of adolescent idiopathic scoliosis. Spine (Phila Pa 1976) 2011;36:825-9.

25. Bastrom TP, Marks MC, Yaszay B, Newton PO; Harms Study Group. Prevalence of postoperative pain in adolescent idiopathic scoliosis and the association with preoperative pain. Spine (Phila Pa 1976) 2013;38:1848-52. 